\title{
Single Dose Antibiotic Prophylaxis in Lumbar Stenosis or Disc Surgery: A Review of 117 Cases
}

\author{
Zohreh Habibi $^{1,2,}$; Zaker saedinejad ${ }^{3}$; Owrang Eilami ${ }^{3}$ \\ ${ }^{1}$ Department of Neurosurgery, Children's Hospital Medical Center, Tehran University of Medical Sciences, Tehran, IR Iran \\ 2 Brain and Spinal Cord Injury Research Center, Tehran, IR Iran \\ 3 Department of Infectious Diseases, Yasuj University of Medical Sciences, Yasuj, IR Iran \\ ${ }^{*}$ Corresponding author:Zohreh Habibi, Department of Neurosurgery, Children's Hospital Medical Center, Tehran University of Medical Sciences, Tehran, IR Iran. Tel: +98-2166920981, \\ Fax:+98-2166930024, E-mail: zohreh_h56@yahoo.com
}

Received: September 26, 2013; Revised: October 19, 2013; Accepted: November 2, 2013

\begin{abstract}
Background: Administration of prophylactic antibiotics for disc surgery is accepted by most surgeons, but no universal protocol exists. Objectives: To determine the safety and effectiveness of single dose preoperative antibiotics alone in preventing wound infections following single level lumbar laminectomy with or without discectomy.

Patients and Methods: We reviewed 117 consecutive patients ( 68 males and 39 females) who underwent single-level lumbar laminectomy and medial facetectomy for lumbar stenosis, with or without discectomy during a ten-month period. Two grams of intravenous cefazolin was administered at the induction of general anesthesia. During the postoperative period, either in hospital or at home, additional antibiotic prophylaxis was not administered. The wounds were inspected on the first day of surgery at the time of indwelling catheter removal,10-14 days after surgery for suture removal, and 4 to 6 weeks after discharge.

Results: Superficial wound redness was detected in 2 patients (1.7\%), which improved with oral antibiotic, and 1 patient (0.85\%) developed discitis which improved with nonsurgical management. None of the patients needed surgical re-exploration for infection or other complications.

Conclusions: The current retrospective study proved that our antibiotic prophylaxis protocol is safe and efficacious. Assuming that a wound infection rate of about $2 \%$ is considered acceptable after a clean spinal operation, a $1.7 \%$ rate for superficial incisional wound infections is promising. Also, as the incidence of discitis following discectomy has been reported to be between $0.75 \%$ and $3.0 \%, 0.85 \%$ rate of discitis in our series seems acceptable.
\end{abstract}

Keywords:Antibiotic Prophylaxis; Intervertebral disc disease; Lumbosacral Region; Wound Infection

\section{Background}

Postoperative wound infection, also known as surgical site infection (SSI), is a troublesome complication of lumbar spine surgeries and can be associated with serious morbidities, mortalities and increase resource utilization (1). Postoperative wound infections are routinely classified as either superficial or deep. Superficial infections involve the dermis and subcutaneous tissues, and are usually recognized as the more common type (2). The rate of postoperative infection in clean neurosurgical procedures is roughly low, with reported frequencies being up to $1 \%$ to $2 \%(3,4)$. Postoperative wound infection in lumbar disc disease with or without stenosis is less frequently seen compared to spinal tumors or thoraco-lumbar traumatic fractures (4). Even so, in the era without prophylactic antibiotics in lumbar disc surgery, the infection rate was as high as $9 \%(5)$. Therefore, administration of prophylactic antibiotics for disc surgery is accepted by most surgeons, but no universal protocol exists. Center for Disease Control and Prevention (CDC) guideline recommends prophylaxis antibiotics only for the day of surgery (6), while the American Society of Health-System Pharmacists (ASHP) recommendation is a single-dose preoperative antibiotic only (7). However, none of these guidelines have been approved as the standard of care.

\section{Objectives}

In this series, we present the results of single dose preoperative antibiotic administration in 117 patients operated on for single level lumbar stenosis or disc extrusion. This is to determine the safety and effectiveness of single dose preoperative antibiotic alone in preventing wound infections following single level lumbar laminectomy with or without discectomy. 
Habibi Z et al.

\section{Patients and Methods}

In this retrospective study, we reviewed the results of single dose prophylaxis protocol in 117 consecutive patients (68 males and 49 females) who underwent singlelevel lumbar laminectomy and medial facetectomy for lumbar stenosis, with or without discectomy, during a ten-month period (between October 2012 and August 2013). Those who underwent multilevel decompression and/or fusion surgery were not included in this study. According to ASHP recommendation, the antimicrobial prophylaxis protocol of a single dose administration of a first generation cephalosporin (cefazolin) was used in this series. Patients with Diabetes mellitus, morbid obesity, prolonged steroid administration, cardiac valve diseases, re-exploration, and history of allergy to betalactams were excluded. Informed consent was obtained before hospitalization. The protocol used in this study was approved by the institutional medical research and ethics committee. All surgical procedures were performed by one neurosurgeon. The follow-up assessments were performed by the same neurosurgeon and an infectious disease specialist in separate settings.

Patients were instructed to take a shower with antimicrobial soap the night before surgery. Skin-shave was performed just before surgery. The number of personnel present in the operating room was limited to one anesthesiologist, one surgeon, one scrub nurse, and one circular nurse. The entrance of additional staff was forbidden. All procedures were performed in prone position, under general anesthesia. For all patients, 2 gr intravenous cefazolin was administered at the induction of general anesthesia. Once in the operating room, the patients had a ten-minute Betadine scrub followed by Betadine paint. Double gloving for the scrub nurse and surgeon was an inherent part of the protocol. The wounds were generously irrigated with saline containing gentamicin at the end of the procedure, and closed drainage systems were applied for all patients. A second intraoperative dose of cefazolin was administered if blood loss exceeded $1500 \mathrm{~mL}$ during the postoperative period. Either in hospital or at home extra prophylactic antibiotic was not administered. Indwelling catheters were removed 24 hours after surgery even in cases of dural tearing.

\subsection{Follow-Up Protocol}

The wounds were inspected on the first day of surgery at the time of indwelling catheter removal, 10-14 days after surgery for suture removal, and 4 to 6 weeks after discharge. The patients were followed for fever, wound redness and edema, purulent discharge from the wound, wound dehiscence with serous discharge, and symptoms of discitis including disabling low back pain in load bearing positions.

\subsection{Statistical Analysis}

The current study was based on a retrospective analysis of filed data, which included patient's age and gender, diagnosis, operating time, signs and symptoms of wound infections as mentioned above, and need of revision surgery for postoperative infection.

\section{Results}

There were 68 males and 49 females, with age ranging between 19 and 76 years (mean; $41.3+/-3.4$ ). Laminectomy and medial fasetectomy was performed in 36 patients for single level stenosis, while 81 cases needed additional discectomy. The mean operation time was 59 minutes (41- 89 minutes). Indwelling catheters were removed on the first day of surgery, and all patients were discharged on the first or second day. However, one patient was re-admitted 3 weeks after surgery because of symptoms and signs of discitis (0.85\%). None of the patients needed surgical reexploration for infection or other complications.

Fever was detected in 7 cases (5.9\%) in the first 24 hours which resolved in all without any pharmacological intervention. One female patient developed UTI and needed extra doses of antibiotic (oral ciprofloxacin, $500 \mathrm{mg}$ bd, for 10 days). Superficial wound redness was detected in 2 patients $(1.7 \%)$ at the time of suture removal which improved by oral coloxacillin ( $500 \mathrm{mg}$ qid, for 5 days). There was no wound drainage or dehiscence for any of the patients.

One male patient developed disabling back pain and severe intermittent buttock spasm 3 weeks after surgery. There was no radicular pain or neurological deficit on physical examination, and straight leg rising test was negative. No fever or leukocytosis was detected (WBC; 6300), but ESR and CRP levels were considerably high. Magnetic resonance imaging revealed enhancement in disc space without epidural abscess or bone enhancement and destruction. Blood culture could not detect any organism. The culture from disc space was not obtained due to its invasive nature and the high rate of negative results in such situations. The patient underwent empirical treatment with bed rest, bracing, intravenous triple antibiotic therapy (meropenem, vancomycin, clindamycin) for bacterial discitis, and dexamethasone for chemical discitis. The symptoms improved within the first 24 hours of treatment and CRP value started to decrease. This clinical course was more in favor of chemical discitis, but the 6-week antibiotic protocol was completed, as there was no way to completely differentiate chemical from bacterial discitis. The patient recovered without any sequelae.

\section{Discussion}

It has been well documented that antibiotics given perioperatively reduce the rate of postoperative wound infections in lumbar disc surgery (8). Since the study by Horowitz and Curtin published in 1975, prophylactic antibiotics 
have been the standard of care for patients undergoing lumbar disc surgery (9). Even if it is generally accepted that prophylactic antibiotics are beneficial in clean spinal surgery, diverse protocols are used in each institute and by each surgeon. Indeed, the timing, duration, and choice of perioperative antibiotics for lumbar disc surgery are still matters of controversies. The problem of antibiotic prophylaxis in spine surgery seems to be relevant in ethical, medical, legal, and economic terms (4).

\subsection{Antibiotic Choice}

The "ideal" antibiotic for prophylaxis of wound infections in clean surgery should have a relatively low cost, limited toxicity, long half-life in both serum and bone, adequate penetration in subcutaneous and muscular tissues and intervertebral disc, and should be sufficiently broad to be effective but limited enough to avoid resistance and superinfections (4). Gram-positive Staphylococci are the most common causative pathogens for postoperative infections (3). A first-generation cephalosporin is the most widely accepted choice in spinal surgery because it is active against staphylococcal species, is relatively nontoxic and inexpensive, and it provides good soft tissue and bone penetration (2). Also, first generation cephalosporin has been proved to be more cost-beneficial compared with other wide-spectrum antibiotics such as Ampicilin-sulbactam (10).

\subsection{Timing}

In most centers, preoperative antibiotics are administered approximately thirty minutes before the actual surgical starting time, preferably at the induction of general anesthesia (4, 8). However, one clinical study confirmed that in humans, antibiotics given in the 2 hours before skin incision had the greatest effect on reducing surgical wound infections (11).

\subsection{Duration}

Until the previous decade, most studies including the study by Horowitz and Curtin, proposed continuation of antibiotics in the postoperative period $(6,9)$. However nowadays, individual protocols vary and range from a single preoperative dose to multiple postoperative doses. The American Society of Health-System Pharmacists (ASHP) recommends only a single-dose of 1000-mg cefazolin at the time of induction of anesthesia for prevention of SSI (7). Some studies have shown that single-dose prophylaxis is as effective as multidose prophylaxis; however, this is not universally accepted $(2,4,8)$.

Kanayama and colleagues have shown that antimicrobial prophylaxis (AMP) protocol based on the CDC guideline did not increase the incidence of postoperative infections in lumbar spine surgery. In this AMP protocol, antibiotics were given only on the day of surgery (12). Khan et al. in a retrospective analysis of 100 cases of lum- bar surgery found that a single preoperative shot of antibiotic is equally effective for SSI prophylaxis compared with multiple doses of antibiotics (13). The investigation by Dobzyniak and colleagues did not demonstrate a statistically significant difference in the rate of infection between patients receiving preoperative antibiotics alone (1.56\%) versus those receiving preoperative followed by postoperative antibiotics (1.20\%) (8). In another retrospective study by Mastronardi et al, intraoperative protocol consisting of a single administration of the compound ampicillin-sulbactam at the induction of anesthesia, with the adjunct of a 1- or 2-dose injection of teicoplanin in instrumented and long procedures, proved to be safe and efficacious (4). Even in patients who underwent spine instrumentation for degenerative conditions, the results of Hellbusch el al. study showed no significant difference in infection rates between the preoperative only protocol and the extended ten-day postoperative protocol (2).

In Iran, specially in private centers, patients often receive extra doses of postoperative prophylactic antibiotics. One study revealed that in private hospitals in Shiraz, Iran, the overall compliance with ASHP guidelines was $10.13 \%$ and about $90 \%$ of patients received inappropriate surgical prophylaxis (14). In another study in Shiraz, the results showed that the direct cost of current surgical antibiotic prophylaxis was approximately 14 folds greater than the cost of a suggested regimen by guideline (3).

\subsection{Detrimental Effects of Prolonged Postoperative Antibiotics}

Most patients who were operated on for lumbar disc disease were stable for discharge either later that day or on the first postoperative day (8). Patients who undergo clean surgical procedures are most likely to be harmed by unjustified use of antibiotics. In most studies mentioned above, the addition of postoperative antibiotics dose did not significantly decrease the infection rate. On the other hand, the unwarranted prolonged use of antibiotics exposes patients to more adverse drug effects, increases the likelihood of developing infections by antibiotic-resistant bacteria, inhibits normalization of body temperature, elevates CRP levels, prolongs the duration of hospital stay, and increases the overall medical cost (15).

The current retrospective study proved that our antibiotic prophylaxis protocol is safe and efficacious. Assuming that a wound infection rate of about $2 \%$ is considered acceptable after a clean spinal operation (4), a 1.7\% rate for superficial incisional wound infections is promising. Also, as the incidence of discitis following discectomy is reported at between $0.75 \%$ and $3.0 \%(16)$, the $0.85 \%$ rate of discitis in our series seems acceptable.

We do not claim that this is a novel work, but it is a local experience of an established protocol (ASHP) in an Iranian hospital. Limited analogous studies have shown that single dose antimicrobial prophylaxis is good enough in lumbar spine surgery $(2,4,8,12,13)$. However, no similar 
study has been performed in Iran, and the authors tried to carry out a standard protocol in a country where based on published data about $90 \%$ of patients receive inappropriate extra doses of surgical prophylaxis (14).

\subsection{Shortcoming and Needs for Further Study}

There are several limitations in the current study; this study had a retrospective character and the sample size was relatively small. To confirm the efficacy of our antibiotic prophylaxis protocol in clean spinal surgery, a randomized prospective controlled clinical trial on a large sample is needed.

\subsection{Conclusions}

The current trend in lumbar disc surgery is for discharge to home the day of surgery or on the first postoperative day. Prolonged prophylactic antibiotic administration may increase hospital stay and side effects. Although some authors affirm that postoperative doses of antibiotics can be administered during the first 24 hours or more, we agree with others who believe that additional doses have no benefit in reducing the incidence of wound infections. Even if the current study has the weakness of a retrospective character, our single dose prophylaxis protocol was shown to be safe and efficacious. This statement seems to be valid even in the presence of closed vacuum drains.

\section{Acknowledgements}

None declared.

\section{Authors' contribution}

Zohreh Habibi; study concept and design, acquisition of data, analysis and interpretation of data, drafting of the manuscript and study supervision. Zaker saedinejad; acquisition of data, analysis and interpretation of data. Owrang Eilami; statistical analysis, administration, technical and material support.

\section{Financial Disclosure}

Authors did not receive any grant or funding. None of the authors have any conflict of interest.

\section{Funding/Support}

There was no source of extra-institutional funding in this study.

\section{References}

1. Yamamoto M, Jimbo M, Ide M, Tanaka N, Umebara Y, Hagiwara S. Perioperative antimicrobial prophylaxis in neurosurgery: clinical trial of systemic flomoxef administration and saline containing gentamicin for irrigation. Neurol Med Chir (Tokyo). 1996;36(6):370-6.

2. Hellbusch LC, Helzer-Julin M, Doran SE, Leibrock LG, Long DJ, Puccioni MJ, et al. Single-dose vs multiple-dose antibiotic prophylaxis in instrumented lumbar fusion-a prospective study. Surg Neurol. 2008;70(6):622-7.

3. Askarian M, Reza Moravveji A, Assadian O. Prescription of prophylactic antibiotics for neurosurgical procedures in teaching hospitals in Iran. Am J Infect Control. 2007;35(4):260-2.

4. Mastronardi L, Tatta C. Intraoperative antibiotic prophylaxis in clean spinal surgery: a retrospective analysis in a consecutive series of 973 cases. Surg Neurol. 2004;61(2):129-35.

5. El-Gindi S, Aref S, Salama M, Andrew J. Infection of intervertebral discs after operation. J Bone Joint Surg Br.1976;58(1):114-6.

6. Kanayama M, Hashimoto T, Shigenobu K, Oha F, Togawa D. Effective prevention of surgical site infection using a Centers for Disease Control and Prevention guideline-based antimicrobial prophylaxis in lumbar spine surgery. J Neurosurg Spine. 2007;6(4):327-9.

7. ASHP Therapeutic Guidelines on Antimicrobial Prophylaxis in Surgery. American Society of Health-System Pharmacists. Am J Health Syst Pharm. 1999;56(18):1839-88.

8. Dobzyniak MA, Fischgrund JS, Hankins S, Herkowitz HN. Single versus multiple dose antibiotic prophylaxis in lumbar disc surgery. Spine (Phila Pa 1976). 2003;28(21):E453-5

9. Horwitz NH, Curtin JA. Prophylactic antibiotics and wound infections following laminectomy for lumber disc herniation. $J$ Neurosurg. 1975;43(6):727-31.

10. Zhu XL, Wong WK, Yeung WM, Mo P, Tsang CS, Pang KH, et al A randomized, double-blind comparison of ampicillin/sulbactam and ceftriaxone in the prevention of surgical-site infections after neurosurgery. Clin Ther. 2001;23(8):1281-91.

11. Classen DC, Evans RS, Pestotnik SL, Horn SD, Menlove RL, Burke JP. The timing of prophylactic administration of antibiotics and the risk of surgical-wound infection. N Engl J Med. 1992;326(5):281-6.

12. Kanayama M, Oha F, Togawa D, Shigenobu K, Hashimoto T Is closed-suction drainage necessary for single-level lumbar decompression?: review of 560 cases. Clin Orthop Relat Res. 2010;468(10):2690-4.

13. Khan IU, Janjua MB, Hasan S, Shah S. Surgical site infection in lumbar surgeries, pre and postoperative antibiotics and length of stay: a case study.JAyub Med Coll Abbottabad.2009;21(3):135-8.

14. Mahdaviazad H, Masoompour SM, Askarian M. Iranian surgeons' compliance with the American Society of Health-System Pharmacists guidelines: antibiotic prophylaxis in private versus teaching hospitals in Shiraz, Iran. J Infect Public Health. 2011;4(5-6):253-9.

15. Ohtori S, Inoue G, Koshi T, Yamashita M, Yamauchi K, Suzuki M, et al. Long-term intravenous administration of antibiotics for lumbar spinal surgery prolongs the duration of hospital stay and time to normalize body temperature after surgery. Spine (Phila Pa 1976). 2008;33(26):2935-7.

16. Housden PL, Sullivan MF. Do augmentin or cefuroxime reach effective levels in lumbar vertebral discs when used prophylactically for discectomy? A preliminary report. Eur Spine J. 1993;2(3):145-8. 